\title{
Mechanical Sequential Counting with Liquid Marbles
}

\author{
Thomas C. Draper ${ }^{1(凶)}[0000-0001-6466-8796]$, Claire \\ Fullarton $^{1[0000-0003-0640-0497]}$, Neil Phillips ${ }^{10000-0002-7878-0196]}$, Ben P. J. \\ de Lacy Costello ${ }^{2[0000-0003-2999-6801]}$, and Andrew \\ Adamatzky ${ }^{10000-0003-1073-2662]}$ \\ 1 Unconventional Computing Laboratory, \\ University of the West of England, Bristol, BS16 1QY, UK \\ \{tom.draper, claire.fullarton, neil.phillips, andrew . adamatzky\}@uwe.ac.uk \\ 2 Institute of Biosensing Technology, Centre for Research in Biosciences, \\ University of the West of England, Bristol, BS16 1QY, UK \\ ben.delacycostello@uwe.ac.uk
}

\begin{abstract}
This paper reports the first working example of a liquid marble-operated sequential binary counting device. We have designed a lightweight gate that can be actuated by the low mass and momentum of a liquid marble. By linking a number of these gates in series, we are able to digitally count up to binary 1111 (upper limit only by our requirements). Using liquid marbles in such a system opens up new avenues of research and design, by way of modifying the coating and/or core of the liquid marbles, and thereby giving extra dimensions for calculation (e.g. a calculation that takes into consideration the progress of a chemical reaction inside a liquid marble). In addition, the new gate design has multiple uses in liquid marble rerouting.
\end{abstract}

Keywords: Liquid marbles, Unconventional computing, Binary counter, Logic gate, Particle-coated droplets, Mechanical computing

\section{Introduction}

\section{$1.1 \quad$ Liquid Marbles}

Liquid marbles (LMs) were first reported by Aussillous and Quéré in 2001 [3], and have since become increasingly popular in chemistry, particularly condensed matter. They are composed of two parts: a microliter sized core of liquid (usually water), surrounded by a powder coating. This gives them their other name 'particle-coated droplets'. A typical volume of a LM is $10 \mu \mathrm{L}$, which results in a typical diameter of $3 \mathrm{~mm}$. A schematic of a LM is shown in Fig. 1. We shall look at both the core and coating in turn.

The bulk of a LM is composed of the core. This microliter droplet is made of water generally (because its high surface tension allows for the easiest LM formation), though glycerol is quite common [4], and even petroleum has been used [7]. This paper will focus on water-filled LMs. 


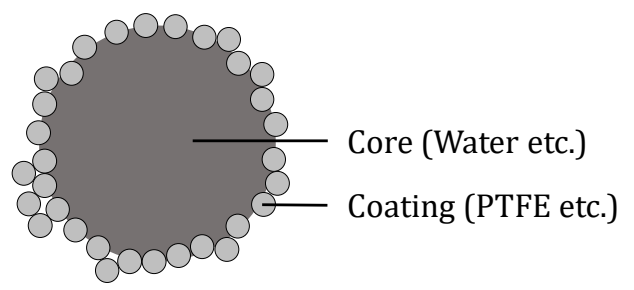

Fig. 1. A schematic of a liquid marble. The core is generally comprised of water or glycerol, and the hydrophobic powder coating could be PTFE, PE, lycopodium grains, etc. A typical diameter of the entire LM is $3 \mathrm{~mm}$, whilst the powder particle sizes could be $10 \mathrm{~nm}$ to $400 \mu \mathrm{m}$ in diameter. Note the non-homogeneous coating.

The coating of a LM is comprised of a micro- or nano-sized powder, that (for water cores) is hydrophobic. 'Hydrophobic' comes from the Ancient Greek 'fear of water'. Chemically, hydrophobic powders are normally lacking in polar intramolecular bonds, which results in few intermolecular hydrogen-bonds forming between the water and the substrate. It is this shortage of attractive forces that is often (mistakenly) portrayed as a water-repelling repulsive force. Common examples of LM powder coatings include polytetrafluoroethylene (PTFE) [5], polyethylene (PE) [1] and modified-lycopodium grains [3]. A variety of possible powder coatings are demonstrated in Fig. 2. Note the difference in particle size, especially in the hybrid example shown in Fig. 2(d). This (in combination with the powders degree of hydrophobicity) gives rise to very different characteristics of LM lifetime, ruggedness and hysteresis. For a recent overview on these dependencies, see reference [11].

As can be seen in Figs. 1 \& 2, the coating of LMs is not homogeneous. Rather, it is a mixture of single-layer and multilayer particles. Whether a singleor multilayer is formed is dependent on the identities of both the core and the coating. Particles with a very high surface contact angle (such as PTFE) tend to form single-layers. Conversely, less hydrophobic particles (such as the PE or nickel) tend to form a multilayer. It can be possible to convert a multilayer LM into a single-layer, by repeated rolling. The excess particles fall off the LM, leaving a single-layer.

There are often gaps in the particle coating, where the surface of the core is exposed to the atmosphere and therefore visible. As (perhaps) anticipated, this is more common in single-layered LMs. As a LM ages however, it looses some of its aqueous core to evaporation, which results in a slight contraction of the shell. This has the effect of closing these exposed sections. One would intuitively anticipate that this would, in turn, reduce the evaporation rate of the LM, however this is not the case [11]. The reasons behind this are still unclear, although a possibility is that the shrinking in pore size causes an increase in the capillary effect. This would result in the water core being closer to the surface, and therefore able to evaporate faster. 


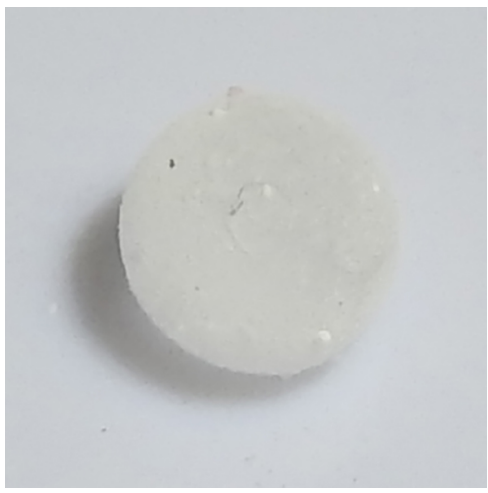

(a)

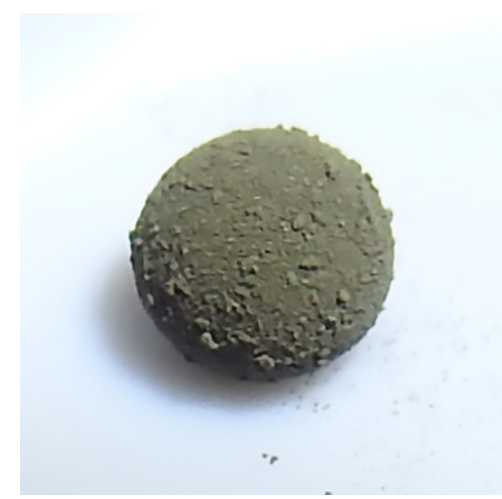

(c)

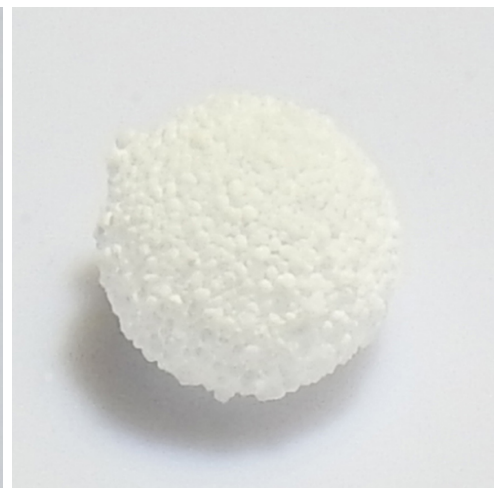

(b)

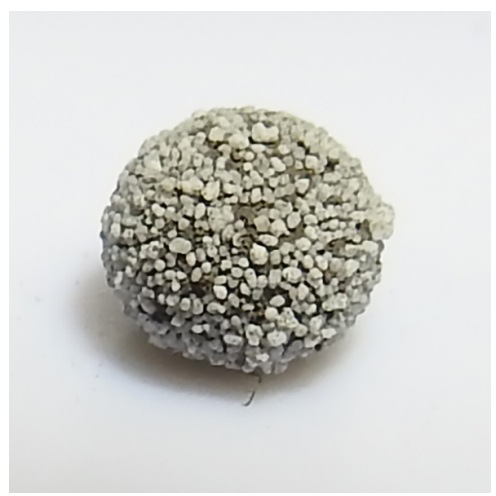

(d)

Fig. 2. Photographs of $10.0 \mu \mathrm{L}$ liquid marbles. All examples have a diameter of $3 \mathrm{~mm}$. The powder coatings portrayed are (a) PTFE (grain size: $6 \mu \mathrm{m}$ to $10 \mu \mathrm{m}$ ), (b) PE (grain size: $100 \mu \mathrm{m}$ ), (c) Nickel (grain size: $4 \mu \mathrm{m}$ to $7 \mu \mathrm{m}$ ) and (d) a Nickel-PE hybrid.

One of the main features of LMs, and one of the reasons for their use in this project, is that they roll with minimal resistance. On a typical surface (e.g. glass), a water droplet will adhere to the surface, causing resistance to its motion. Conversely, if a water droplet is placed on a hydrophobic surface (e.g. a nonstick frying pan), the water droplet will bead up and roll off with ease. A LM is literally coated in a hydrophobic powder, generating a very large contact angle, and therefore rolls with extreme ease. This gives LMs great merit in fields as diverse as glue delivery systems [8] and digital microfluidic bioassays [18].

Liquid marbles are a new, but strong, player in the field of digital microfluidics [17]. Microfluidics involves the formation, behavior, and ultimately the control of microliter quantities of fluid. It is a multidisciplinary area, with a large and growing interest in automation and high-throughput screening. Digital microfluidics is when the fluid is in discrete droplets, as oppose to in a continuous 
flow. There are a number of ways to manipulate these droplets, the most common of which are magnetic [21], electrowetting on dielectric (EWOD) [10], and surface acoustic wave (SAW) [13]. All of these techniques, however, require both a pre-treated surface and electricity. By encapsulating the droplet in a particle coating and forming a LM, there is the potential to remove both of these limitations. This would allow for the construction of cheap devices for point-of-care diagnostics in low-resource areas [20].

In order for the microfluidic LMs to increase in capability, there is a requirement for behavioral control. Control units will need to be able to route [19], merge [4], divide [6], and auto-generate [9] LMs. This work demonstrates a new re-routing technique for LMs, in the form of a mechanical computing device. This device does not require surface-pretreatment, and is powered by gravity: thereby removing the limitations of other digital microfluidic manipulation techniques.

\subsection{Existing Computational Techniques with Liquid Marbles}

Liquid marbles have recently been used experimentally for computation, in a collision-based interaction gate [9]. In the design, two LMs rolled down slopes towards each other and collided. The resulting change in vector was then interpreted as computation. In this LM interaction gate, the Boolean-determining signal was the presence (TRUE) or absence (FALSE) of a LM. It was noted that, due to the soft shell-like nature of the LMs, the collisions observed Margolus pathways [15], as shown in Fig. 3(a). These differ from the better-known billiardball pathways by allowing for the finite amount of time it takes for a soft-sphere to deform. This analogy could be broken, however, by increasing the kinetic energy of the impact above the effective surface tension of the LM. Above this threshold, the LMs would coalesce, and the system acted like the fusion gate shown in Fig. 3(b). In both instances the gate implemented AND and AND-NOT logical functions, based on the location of output LMs.

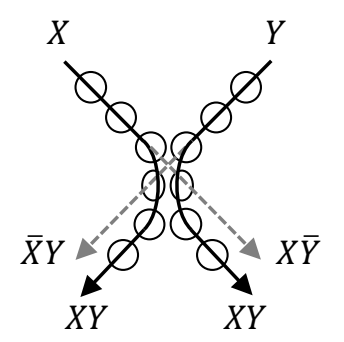

(a)

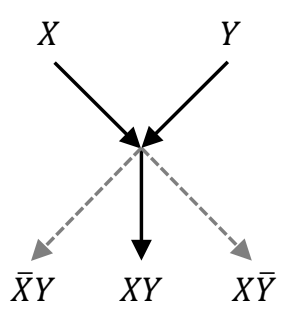

(b)

Fig. 3. Diagrams of interaction gates. (a) A Margolus gate, demonstrated using compressible spheres as signals. (b) A fusion gate, where the two signals coalesce to form one new signal. 
The LM coating used in [9] was a mix of nickel $(\mathrm{Ni})$ and $\mathrm{PE}$, forming a hybrid LM that was ferromagnetic enough to be held by electromagnets. This feature was used to enable accurate timings on the interaction gate. Without this feature, ensuring timings and collisions was intractable.

Recent work [11] has shown that the impact survival time of PE LMs is far superior to Ni-PE hybrid LMs. Unfortunately, the non-magnetic nature of PE makes precise synchronization of the LMs arduous. It was decided, therefore, to develop a computing device that did not require such accurate timings, but could instead be run sequentially.

\section{Liquid Marbles for Mechanical Counting}

\subsection{Mechanical Flip-Flop Gates}

Mechanical flip-flops have been designed previously, though they have always been actuated by something relatively heavy, such as a coin [2], a ball [16], or even a can [14]. We have developed a distinct and notably different mechanical flipflop, that is designed specifically to be actuated by the low mass and momentum of a single LM.

Shown in Fig. 4(a), the flip-flop is roughly shaped like an isosceles-triangle, with the top pointing to either 10 o'clock or 2 o'clock (its two bistable positions, when compared to the hour hand on the face of a clock). These are visualized in Fig. 4. When in use, LM \#1 approaches the flip-flop from the top and follows the path guided to it by the flip-flop. As the LM moves along, its mass causes the flip-flop to 'flip'. As a result, when LM \#2 approaches the flip-flop, it will both be guided in the other direction and reset the flip-flop to its original position. LM \#3 will then follow the same path as LM \#1, and so on. There are two overall consequences of this: as each LM passes through, the resting position of the flip-flop oscillates between the two bistable positions - 10 o'clock and 2 o'clock; additionally, alternate LMs exit through alternate pathways. These pathways are demonstrated in Fig. 4(b).

This design acts like a traditional electronic flip-flop: it is a bistable multivibrator, and each of its two positions can be interpreted as binary 0 or 1 . The rolling LMs act in a similar way to the electronic data signal pulses, changing the reading as they arrive. The LMs roll by converting their potential energy into kinetic energy, and so the system is powered by gravity instead of electricity. The LMs roll along guides, which act like the wires that guide electrons in an electronic system.

Each flip-flop has been laser cut from $3 \mathrm{~mm}$ thick cast acrylic. It has dimensions of $29 \mathrm{~mm} \times 14 \mathrm{~mm} \times 3 \mathrm{~mm}$, and weighs $154 \mathrm{mg}$. The pivot point (diameter: $0.60 \mathrm{~mm}$ ), easily seen in Fig. 4(a), has been engineered to be at the center of mass of the flip-flop.

\subsection{Liquid Marble Actuated Mechanical Counter}

By linking four of our flip-flop gates in series, we were able to design and construct a proof-of-principle logic device, capable of counting up to binary 1111 


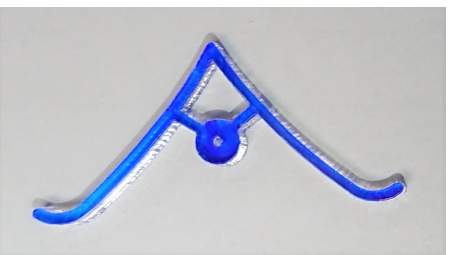

(a)

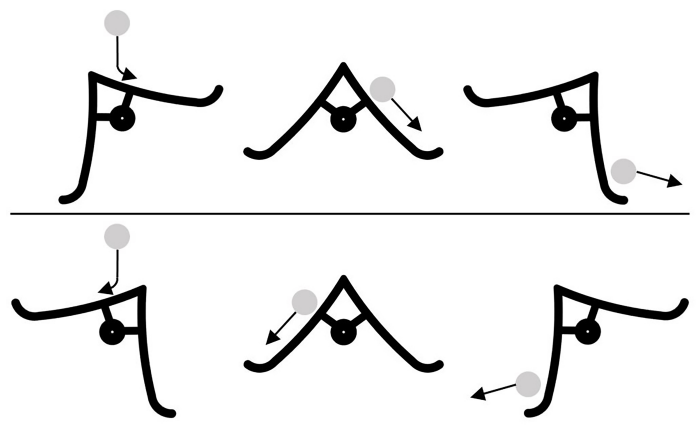

(b)

Fig. 4. (a) A photograph of our flip-flop gate. The front has been colored for visibility. The flip-flop is $29 \mathrm{~mm}$ across. (b) A diagram showing the motion that our mechanical flip-flop gate takes, when actuated using a LM. Starting at the 10 o'clock position, the LM enters the system and interacts with the flip-flip - rotating the flip-flop to the 2 o'clock position whilst the LM exits to the right. If at the 2 o'clock position when the LM enters, the flip-flop is rotated to the 10 o'clock position, and the LM exits to the left. A typical LM has a diameter of $3 \mathrm{~mm}$.

or decimal 15. The basic principle is similar to part of a 1965 patent [12]. Our design schematic is shown in Fig. 5(a) and a photograph of the constructed device is shown in Fig. 5(b). In this design, a flip-flop is considered to represent binary 0 when pointing to 10 o'clock, and binary 1 when pointing to 2 o'clock. The memory is read from the bottom up. So decimal 8 would be binary 1000, with the 1 physically situated at the bottom of the device.

To start, the system should have a clear memory, with all the flip-flops pointing to 10 o'clock, and the readout being 0000 . When LM \#1 enters the system from the top, it queries the first flip-flop and obeys the logic table shown in Tab. 1. On discovering that the first flip-flop reads 0 , it changes it to a 1 and exits to the right (exiting the system). At this point the readout is 0001. One can consider that the LM has queried the memory, added one to its value, then rewritten the new value back to the memory: a destructive readout.

Table 1. Logic rules for the liquid marbles to observe, as they pass through the mechanical counting device and interact with flip-flop gates.

\begin{tabular}{ccl}
\hline Flip-Flop & Bit & \multicolumn{1}{c}{ Action } \\
\hline Left & 0 & Change bit to 1, exit system \\
Right & 1 & Change bit to 0, query next bit (or OvERFLow if none) \\
\hline
\end{tabular}

When LM \#2 next enters the system, it queries the first flip-flop and reads a 1 , so it changes it to a 0 and exits to the left towards the second flip-flip. Here 


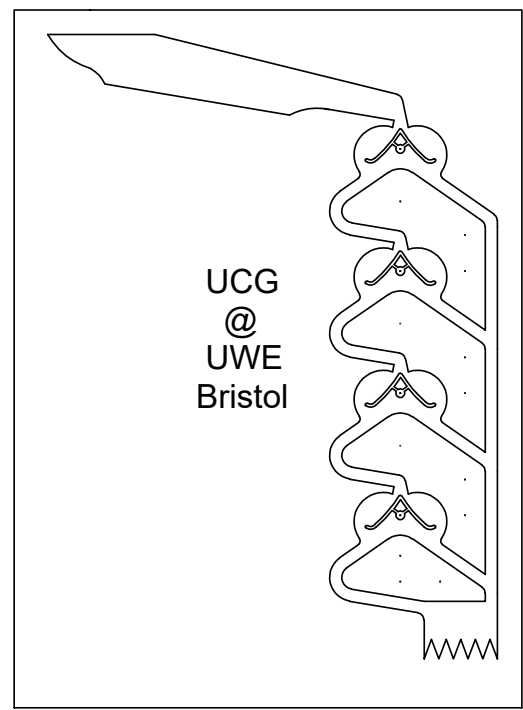

(a)

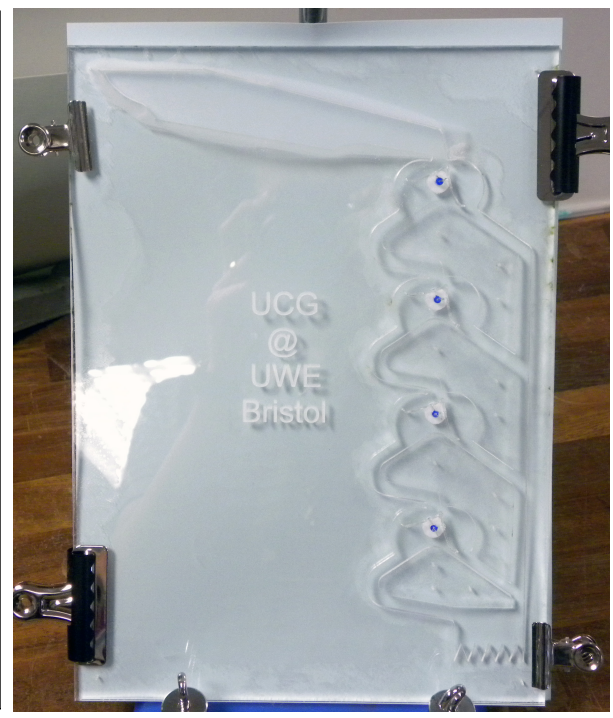

(b)

Fig. 5. The (a) CAD design file and (b) a photograph of the LM counting device. It measures $208 \mathrm{~mm} \times 286 \mathrm{~mm} \times 6 \mathrm{~mm}$.

it reads a 0 , so changes it to a 1 and exits to the right (exiting the system). At this point the readout is 0010 . This continues in a similar manner until the system reads 1111 (decimal 15), at which point the next LM will cause a memory overflow and reset the readout to 0000 . Stills from a video portraying operation of the device can be seen in Fig. 6. A more abstract view of the devices operation can be envisioned, where the two different bistable positions are indicated by a headless arrow. As a LM moves through the system it causes the flip-flop gates to alternate position, with 16 total possible states before overflow occurs $\left(2^{4}=16\right)$. Such a schematic, with all possible states portrayed in order, reading from left to right \& top to bottom, can be seen in Fig. 7 .

As the LMs move through the device, it is worthy of note that they do not slide. Instead, the LMs demonstrate a superposition of both rotational and translational motion (i.e. rolling). This is in direct contrast to the motion expected of uncoated droplets. A side effect of this superposition is observed when two LMs are permitted to roll next to each other. Rather than running together like smooth ball bearings, they instead bounce off each other. This is caused by the approaching front of the chasing LM moving vertically down, in direct contrast to the rear of the leading LM moving vertically up. The clash causes both LM to temporally pause, and for the chasing LM to actually roll backwards. As such, the LMs must be timed so that they do not make contact with each other.

The LMs used in the counting device had a core of pure deionized water, with a volume of $15.0 \mu \mathrm{L}$. We found that this volume had the optimal mass to actuate 


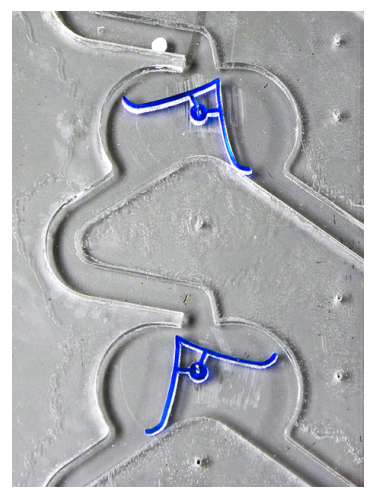

(a)

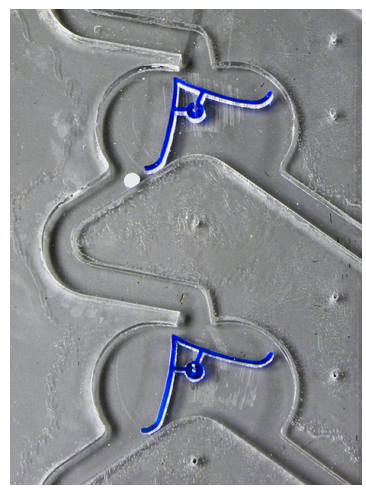

(d)

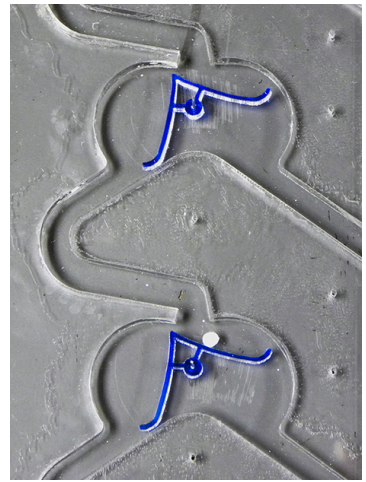

(g)

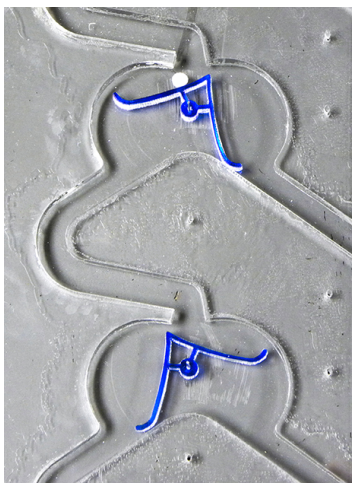

(b)

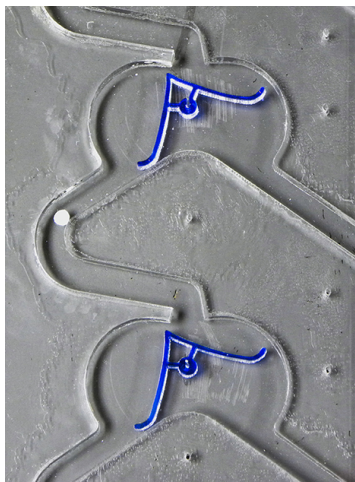

(e)

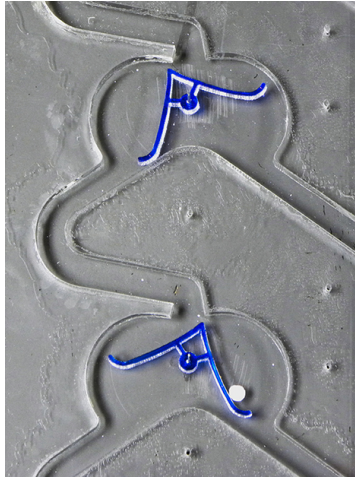

(h)

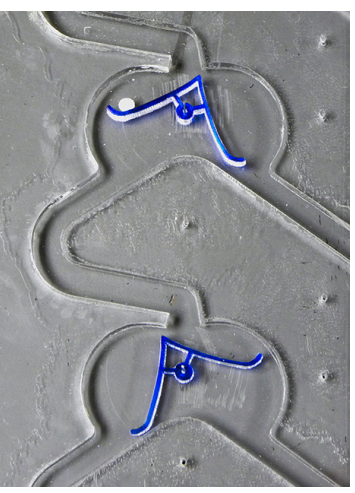

(c)

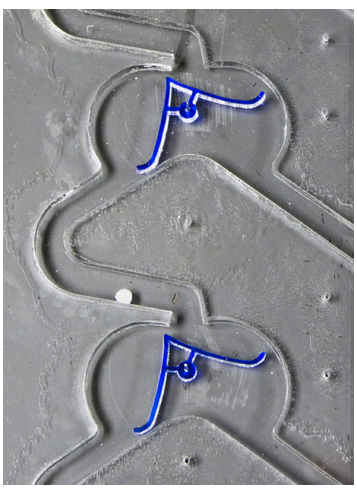

(f)

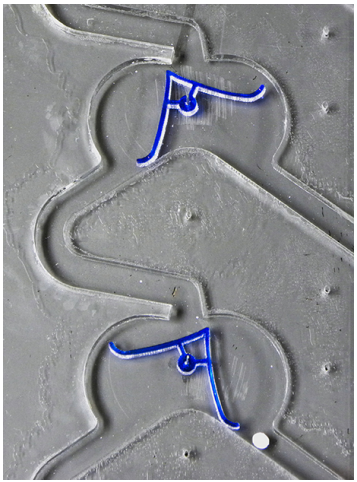

(i)

Fig. 6. Stills from a video demonstrating the counting device. These were restaged to improve clarity. Originals and video are available. The LM approaches from the top, flips the first gate from right to left, exits to the left, then flips the second gate from left to right, then exits to the right. The respective relative times of the frames are $0 \mathrm{~ms}, 99 \mathrm{~ms}, 233 \mathrm{~ms}, 466 \mathrm{~ms}, 533 \mathrm{~ms}, 633 \mathrm{~ms}, 733 \mathrm{~ms}, 1033 \mathrm{~ms}$ and $1199 \mathrm{~ms}$. 

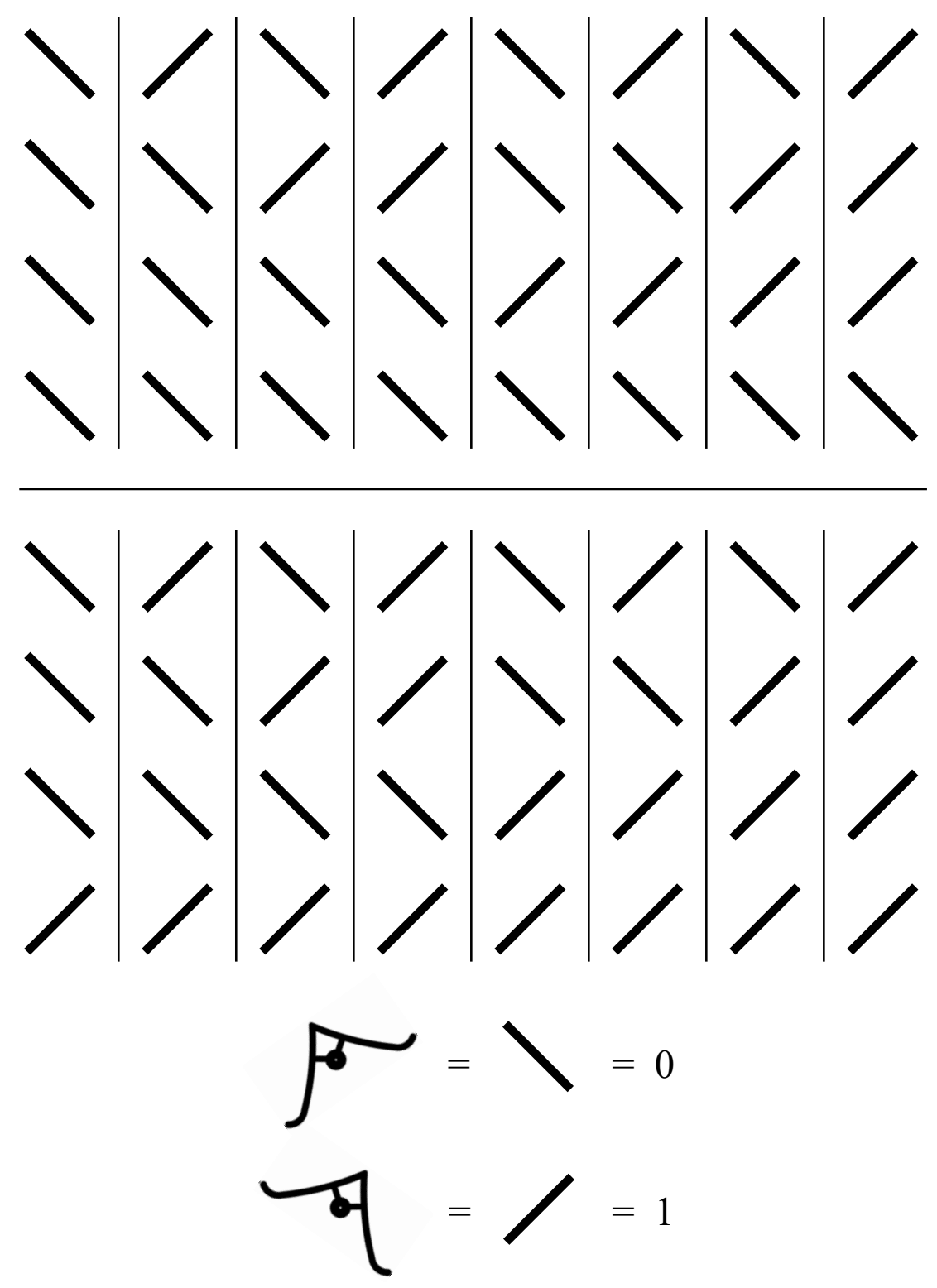

Fig. 7. An abstract schematic showing the 16 different positions the flip-flop gates take as each LM moves through the system. Read from left to right \& top to bottom. Flip-flops are grouped in fours; each group represents the processing of a single LM. The least significant bit is at the top of a group, and the most significant bit is at the bottom of each group. On the $16^{\text {th }} \mathrm{LM}$, the device overflows and assumes the first position (i.e. binary 0000). 
our flip-flop gates. Heavier LMs often deformed or got stuck, whilst lighter LMs were not able to actuate the gates. We used two different coatings for the LMs. The first was a Ni-PE coating, originally portrayed in reference [9]. The second was a pure PE coating, which has recently been shown to make stronger and longer-lasting LMs in collision-based activities [11]. LMs made from either of these coatings were able to successfully traverse our counting device.

\subsection{Speed-Bumps in the Design Process}

As with any $R \& D$ adventure, throughout the design of the counting device there were obstructions to overcome. We hope that this section will help anyone who intends to repeat or continue this work.

The main difficultly we had to overcome was the design and construction of a mechanical flip-flop that could be actuated by the small weight of a LM ( $\sim 16 \mathrm{mg}$, less than two grains of sand). This was achieved over multiple iterations of constructing, testing and optimizing. During this process, we concentrated on minimizing the mass of the gate (while maintaining structural integrity), as this would reduce the moment of inertia. This had to be balanced with the limitations of the equipment available to us. The flip-flops were laser cut from $3 \mathrm{~mm}$ acrylic, and as such were limited by the both the melting of the acrylic and the Gaussianstyle beam of the laser. Consequently, the absolute smallest arm-width we could cut was $0.8 \mathrm{~mm}$, and the smallest we could reliably cut was $1.0 \mathrm{~mm}$ (which we used in the device).

The design also had to balance the increased moment of inertia caused by having long 'arms', with the increased pivoting ratio. This was achieved through testing a series of different sizes. During these tests, the pivot location was constantly updated to remain at the center of mass, therefore minimizing the force required to rotate the gate.

It was necessary to minimize the friction around the pivot. We initially used PTFE coated wire as the pivot (PTFE is regarded as one of the lowest-friction materials). However, the PTFE coating required a reduction in the diameter of the metal core of the wire. This had the unfortunate side effect of making the pivot too flexible. Instead, a steel pivot was used, which also has a low friction coefficient. However, this was insufficient, and so we laser-cut our own washers from $0.25 \mathrm{~mm}$ PTFE sheets (we were unable to source a supplier to provide them small enough). This proved to reduce the friction sufficiently to allow the flip-flop to rotate around the pivot.

The pitch of the device is critical to its successful operation. If the angle is too steep, then the LM will run too fast and either mis-actuate the flip-flop or come off the device entirely. Likewise, if the angle is too gentle, the LM will either not have enough momentum to actuate the flip-flop, or it will not roll at all. We discovered, using an in-house rig to adjust and accurately measure the pitch, that the ideal angle for our device is $52^{\circ}$ from horizontal for $15 \mu \mathrm{L}$ LMs. 


\subsection{Materials and Design}

Both the flip-flop gates and the mechanical counting device were designed using the CAD software Autodesk AutoCAD 2018. They were then laser cut from $3 \mathrm{~mm}$ clear cast acrylic. The counting device was made up of a backboard and a front-board, which was itself composed of a large piece and several smaller pieces. The separate components of the counting device were held together by pins and clamps, before being affixed using RS Pro AB-3 Acrylic Adhesive (RS Components). Steel pins (0.50 mm diameter) were installed as pivots, and glued in position using epoxy resin. The PTFE washers were also CAD designed (outer diameter: $10.0 \mathrm{~mm}$, inner diameter: $0.6 \mathrm{~mm}$ ), before being laser cut from $0.25 \mathrm{~mm}$ sheet PTFE. The washers were then placed onto the steel pins before the flip-flop gates. For optimal performance, our device was tilted at $52^{\circ}$ from horizontal.

\section{Conclusions and Future Work}

This paper demonstrates the first sequential logic device implemented using LMs. Through the careful design and construction of a light-weight and low-friction flip-flop gate, a simple proof-of-principle counting device has been constructed. This device counts upwards in integers, from binary 0000 to 1111 (decimal 0 to 15). This upper limit is only restricted by the size of the constructed device, leaving the possibility for much larger devices.

We have also reported on the design and construction of a new microfluidic LM router: the small mechanical LM-actuated flip-flop. By using gravity to power the device, and forgoing the traditional surface pre-treatment, development of low-resource devices is possible. There is also scope for using the LMs as cargo-carriers, and having the flip-flop gates act as path-directors.

A possible use for a device like this is in patient-care in challenging environments. If a spring-loaded syringe pump was injecting into a patient, then a small side-branch could be taken off the line. This spur (diverting less than 1\%) could form LMs (using the set-up reported in reference [9]) which run through the counting system. This would provide a clear non-electrical digital readout of how much has been injected, compared to the analogue readout of the syringe. However, this set-up would obviously require pre-calibration.

There is much scope for continued worked in this field. We are already designing a larger and more complex arithmetic machine, to fully take advantage of the flip-flop gates. Additionally, the use of LMs allows for an entirely new dimension of programming: the LMs represent a combination of data signals and a clock pulse, and by varying the coating and/or core of the LM, each can be given a different purpose and identity. There is also scope for chemical reactions to be undertaken inside the LMs: for example a reaction that destroys a LM after a certain amount of time could be used to provide a time-limit on data signals.

In summary, we have produced a working model of a LM-actuated sequential binary counting device. This proof-of-principle device has enormous scope for continued development, and we anticipate a variety of designs in the future. 
Acknowledgements. This research was supported by the EPSRC with grant EP/P016677/1 'Computing with Liquid Marbles'.

\section{References}

1. Asare-Asher, S., Connor, J.N., Sedev, R.: Elasticity of liquid marbles. J. Colloid Interface Sci. 449, 341-346 (2015). https://doi.org/10.1016/j.jcis.2015.01.067

2. Asbury, W.: US Patent 957,135 (1909)

3. Aussillous, P., Quéré, D.: Liquid marbles. Nature 411(6840), 924-927 (2001). https://doi.org/10.1038/35082026

4. Aussillous, P., Quéré, D.: Properties of liquid marbles. Proc. R. Soc. A Math. Phys. Eng. Sci. 462(2067), 973-999 (2006). https://doi.org/10.1098/rspa.2005.1581

5. Bhosale, P.S., Panchagnula, M.V., Stretz, H.A.: Mechanically robust nanoparticle stabilized transparent liquid marbles. Appl. Phys. Lett. 93(3), 034109 (2008). https://doi.org/10.1063/1.2959853

6. Bormashenko, E., Bormashenko, Y.: Non-Stick Droplet Surgery with a Superhydrophobic Scalpel. Langmuir 27(7), 3266-3270 (2011). https://doi.org/10.1021/la200258u

7. Bormashenko, E., Pogreb, R., Balter, R., Aharoni, H., Aurbach, D., Strelnikov, V.: Liquid marbles containing petroleum and their properties. Pet. Sci. 12(2), 340-344 (2015). https://doi.org/10.1007/s12182-015-0016-y

8. Chandan, S., Ramakrishna, S., Sunitha, K., Satheesh Chandran, M., Santhosh Kumar, K.S., Mathew, D.: pH-responsive superomniphobic nanoparticles as versatile candidates for encapsulating adhesive liquid marbles. J. Mater. Chem. A 5(43), 22813-22823 (2017). https://doi.org/10.1039/C7TA07562F

9. Draper, T.C., Fullarton, C., Phillips, N., de Lacy Costello, B.P., Adamatzky, A.: Liquid marble interaction gate for collision-based computing. Mater. Today 20(10), 561-568 (2017). https://doi.org/10.1016/j.mattod.2017.09.004

10. Fair, R.B.: Digital microfluidics: is a true lab-on-a-chip possible? Microfluid. Nanofluidics 3(3), 245-281 (2007). https://doi.org/10.1007/s10404-007-0161-8

11. Fullarton, C., Draper, T.C., Phillips, N., Mayne, R., de Lacy Costello, B.P.J., Adamatzky, A.: Evaporation, Lifetime, and Robustness Studies of Liquid Marbles for Collision-Based Computing. Langmuir 34(7), 2573-2580 (2018). https://doi.org/10.1021/acs.langmuir.7b04196

12. Godfrey, J.T.: US Patent 3,390,471 (1965)

13. Guttenberg, Z., Müller, H., Habermüller, H., Geisbauer, A., Pipper, J., Felbel, J., Kielpinski, M., Scriba, J., Wixforth, A.: Planar chip device for PCR and hybridization with surface acoustic wave pump. Lab Chip 5(3), 308-317 (2005). https://doi.org/10.1039/B412712A

14. Kimball, W.D., Braren, C.I., Schaefer, G.P.: US Patent 2,052,513 (1930)

15. Margolus, N.: Universal cellular automata based on the collisions of soft spheres. In: Adamatzky, A. (ed.) Collision-based computing, pp. 107-134. Springer (2002)

16. McEvoy, G.N.: US Patent 884,605 (1905)

17. Nguyen, N.T., Hejazian, M., Ooi, C., Kashaninejad, N.: Recent Advances and Future Perspectives on Microfluidic Liquid Handling. Micromachines 8(6), 186 (2017). https://doi.org/10.3390/mi8060186

18. Oliveira, N.M., Reis, R.L., Mano, J.F.: The Potential of Liquid Marbles for Biomedical Applications: A Critical Review. Adv. Healthc. Mater. 6(19), 1700192 (2017). https://doi.org/10.1002/adhm.201700192 
19. Ooi, C.H., Nguyen, N.T.: Manipulation of liquid marbles. Microfluid. Nanofluidics 19(3), 483-495 (2015). https://doi.org/10.1007/s10404-015-1595-z

20. Zhang, Y., Nguyen, N.T.: Magnetic digital microfluidics âĂŞ a review. Lab Chip 17(6), 994-1008 (2017). https://doi.org/10.1039/C7LC00025A

21. Zhang, Y., Park, S., Liu, K., Tsuan, J., Yang, S., Wang, T.H.: A surface topography assisted droplet manipulation platform for biomarker detection and pathogen identification. Lab Chip 11(3), 398-406 (2011). https://doi.org/10.1039/C0LC00296H 